\title{
A GENERALIZATION OF $F$-SPACES AND SOME TOPOLOGICAL CHARACTERIZATIONS OF GCH
}

BY

\author{
MARY ANNE SWARDSON
}

\begin{abstract}
Several topological characterizations involving $F$-spaces of the continuum hypothesis are due to R. G. Woods and E. K. van Douwen. We extend this work by defining a space $X$ to be an $F_{\alpha}$-space if the union of $<\alpha$ cozero-sets is $C^{*}$-embedded in $X$ and by giving, for every infinite cardinal $\alpha$, topological characterizations involving $F_{\alpha}$-spaces of the cardinal equality $2^{\alpha}=\alpha^{+}$.
\end{abstract}

Topological characterizations of the continuum hypothesis $(\mathrm{CH})$ abound in the theory of $F$-spaces. The two which are important for this paper are due to Woods $\left(\left[\mathbf{W o}_{1}\right]\right.$ and $\left.\left[\mathbf{W o}_{2}\right]\right)$ and to van Douwen $\left[\mathbf{v D}_{1}\right]$. We state these as follows:

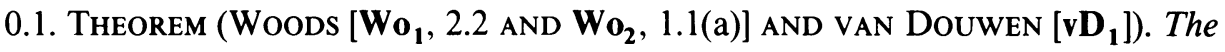
following are equivalent:

(a) $\mathrm{CH}$.

(b) Every small F-space is weakly Lindelöf.

(c) Every small normal countably compact F-space is compact.

Topological characterizations of $\mathrm{CH}$ involving $F$-spaces have also been given by Dow [Do, 3.4], and van Douwen has pointed out a number of others in [ $\mathbf{V D}_{1}$ ]. (We give another such characterization of $\mathrm{CH}$ in this paper (see 3.19 and 5.3(b)).)

The present study, which comes from the author's Ph.D. thesis at Ohio University (done under the direction of R. L. Blair, to whom we are greatly indebted), and which is summarized in [ $\left.\mathbf{S w}_{3}\right]$, has as its purpose an extension of the work of Woods and of van Douwen. We define " $F_{\alpha}$-spaces" and give, for every infinite cardinal $\alpha$, topological characterizations involving $F_{\alpha}$-spaces of the segment $2^{\alpha}=\alpha^{+}$of the generalized continuum hypothesis $(\mathrm{GCH})$.

1. Definitions and conventions. By a topological space (or simply a space), we always mean a Tychonoff space.

Let $X$ be a topological space. Then $C(X)$ (resp. $\left.C^{*}(X)\right)$ denotes the set of all real-valued (resp. bounded real-valued) continuous functions on $X$. For $f \in C(X)$, $Z(f)=\{x \in X: f(x)=0\}$ is a zero-set of $X$. The complement in $X$ of a zero-set of $X$ is a cozero-set of $X$. A subset $A$ of $X$ is $C$-embedded (resp. $C^{*}$-embedded) in $X$ if

Received by the editors October 25, 1982.

1980 Mathematics Subject Classification. Primary 54A35, 54G05, 54D35, 04A30; Secondary 03E10, 54C45, 54D40.

Key words and phrases. $F_{\alpha}$-spaces, $\alpha$-basically disconnected, $\alpha$-pseudocompact, $2^{\alpha}=\alpha^{+}, \chi_{\alpha^{-r e m o t e}}$ point, $\alpha$-open, $\alpha$-closed, almost compact, weak Lindelöf number. 
every $f \in C(A)$ (resp. every $f \in C^{*}(A)$ ) has a continuous extension over $X$. A space $X$ is an $F$-space if every cozero-set of $X$ is $C^{*}$-embedded in $X$ [GJ, 14.25].

What we intend to do in this paper is to add a cardinal parameter $\alpha$ to some of the properties mentioned in 0.1 in such a way that the Woods and van Douwen characterizations of $\mathrm{CH}$ become corollaries of our results in the case $\alpha=\omega$. To this end we make the following definitions: A subset $A$ of a space $X$ is a $G_{\alpha}$-set in $X$ if $A=\cap \mathscr{Q}$, where $\mathscr{U}$ is a collection of open sets in $X$ with $|\mathscr{U}|<\alpha$. A set $A \subset X$ is $G_{\alpha}$-dense in $X$ if every $G_{\alpha}$-set in $X$ meets $A$. $A$ is $\alpha$-open in $X$ if $A=\cup \mathscr{Q}$ where $\mathscr{Q}$ is a collection of cozero-sets of $X$ with $|थ|<\alpha$, and $A$ is $\alpha$-closed in $X$ if $X-A$ is $\alpha$-open in $X$. Clearly finite unions of $\alpha$-open sets are $\alpha$-open, and $A$ is a $\alpha$-closed if and only if $A=\cap \mathscr{Z}$ where $\mathscr{Z}$ is a collection of zero-sets of $X$ with $|\mathscr{Z}|<\alpha$. It is obvious that an $\omega_{1}$-open set is a cozero-set, that an $\omega_{1}$-closed set is a zero-set and that, for $\alpha \geqslant \omega_{1}$, an $\alpha$-closed set is a $G_{\alpha}$-set.

A subset $A$ of $X$ is $z$-embedded in $X$ if every zero-set of $A$ is of the form $Z \cap A$ for some zero-set $Z$ of $X$. Clearly, if $A$ is $z$-embedded in $X$ and if $G$ is $\alpha$-open (resp. $\alpha$-closed) in $A$, then $G=G^{\prime} \cap A$ for some $\alpha$-open (resp. $\alpha$-closed) subset $G^{\prime}$ of $X$.

A space $X$ is an $F_{\alpha}$-space if every $\alpha$-open subset of $X$ is $C^{*}$-embedded in $X$, and $X$ is $\alpha$-basically disconnected if every $\alpha$-open set in $X$ has open closure in $X . X$ is extremally disconnected if every open set in $X$ has open closure in $X$. Clearly $X$ is an $F_{\omega_{1}}$-space if and only if $X$ is an $F$-space, and $X$ is $\omega_{1}$-basically disconnected if and only if $X$ is basically disconnected.

1.1. Remarks. The terms " $F_{\alpha}$-space" and " $\alpha$-basically disconnected space" are used by other authors in senses different from the foregoing:

(a) In [CN, §14], Comfort and Negrepontis restrict attention to the class $\beta$ of spaces that have a base of closed-and-open sets. For $X \in \Re$ and $G$ open in $X, G$ is of type $<\alpha$ if $G$ is the union of $<\alpha$ closed-and-open subsets of $X$. Then, in the sense of [CN], a space $X \in \Re$ is " $\alpha$-basically disconnected" if every open subset of $X$ of type $<\alpha$ has open closure in $X$, and $X$ is an " $F_{\alpha}$-space" if every open subset of $X$ of type $<\alpha$ is $C^{*}$-embedded in $X$ [CN, pp. 350, 343]. It is clear that if $X \in \mathscr{B}$ and if $X$ is an $F_{\alpha}$-space in our sense, then $X$ is an $F_{\alpha}$-space in the sense of [CN]. Our definition of $F_{\alpha}$-space is chosen to insure that our theorems reduce to results about $F$-spaces for the case $\alpha=\omega_{1}$.

(b) In [NL], Neville and Lloyd define a (compact) space $X$ to be an " $F_{\alpha}$-space" if any two disjoint $\alpha$-open subsets of $X$ have disjoint closures in $X$. It can be shown that, in the presence of normality, the Neville and Lloyd definition of $F_{\alpha}$-space coincides with ours, and that, if $X$ is compact and $X \in \mathscr{B}$, then all three definitions of an $F_{\alpha}$-space (that of [CN], of [NL], and ours) coincide.

We shall need the following cardinal functions:

$L(X)=\min \{\kappa$ : every open cover of $X$ has a subcover of cardinality $\leqslant \kappa\}+\omega$.

$w L(X)=\min \{\kappa:$ if $\mathscr{U}$ is an open cover of $X$, then there exists $\mathcal{V} \subset \mathcal{Q}$ such that $|\mathcal{V}| \leqslant \kappa$ and $\cup \mathcal{V}$ is dense in $X\}+\omega$. We say that $X$ is weakly Lindelöf if $w L(X)=\omega$. $d(X)=\min \{|S|: S$ is a dense subset of $X\}+\omega$. $w(X)=\min \{|\Re|: \mathscr{B}$ is a base for $X\}+\omega$. 
For $A \subset X$, define

$\chi(A, X)=\min \{|\Re|: \Re$ is a base for the neighborhoods of $A$ in $X\}+\omega$.

$\psi(A, X)=\min \{|\mathscr{Q}|: \mathcal{Q}$ is a collection of open subsets of $X$ and $A=\cap \mathscr{Q}\}+\omega$.

$\chi_{c}(X)=\sup \{\chi(A, X): A$ is closed in $X\}$.

$\chi_{n z}(X)=\sup \{\chi(A, X): A$ is a nowhere dense zero-set of $X\}$.

2. Properties of $F_{\alpha}$-spaces and $\alpha$-basically disconnected spaces. In proving some elementary results about $F_{\alpha}$-spaces and $\alpha$-basically disconnected spaces, we will frequently make use of the following proposition.

2.1. Proposition. If $G$ is $\alpha$-open in $X$ and if $Z_{1}$ and $Z_{2}$ are disjoint zero-sets of $G$, then $Z_{1}$ and $Z_{2}$ are contained in disjoint $\alpha$-open subsets of $X$.

Proof. Let $G=\cup_{\xi<\kappa} P_{\xi}$, where each $P_{\xi}$ is a cozero-set of $X$ and $\kappa<\alpha$, and let $Z_{1}$ and $Z_{2}$ be disjoint zero-sets of $G$. There exist disjoint cozero-sets $Q_{1}$ and $Q_{2}$ of $G$ with $Z_{1} \subset Q_{1}$ and $Z_{2} \subset Q_{2}$. For each $\xi<\kappa, Q_{1} \cap P_{\xi}$ and $Q_{2} \cap P_{\xi}$ are disjoint cozero-sets of $P_{\xi}$ and hence of $X$ [En, 2.1.B(c)], and thus $\cup_{\xi<\kappa}\left(Q_{1} \cap P_{\xi}\right)$ and $\cup_{\xi<\kappa}\left(Q_{2} \cap P_{\xi}\right)$ are disjoint $\alpha$-open subsets of $X$ containing $Z_{1}$ and $Z_{2}$ respectively.

2.2. Proposition. If $X$ is a space, then the following are equivalent:

(a) $X$ is extremally disconnected.

(b) $X$ is $\alpha$-basically disconnected for every infinite cardinal $\alpha$.

(c) $X$ is an $F_{\alpha}$-space for every infinite cardinal $\alpha$.

Proof. (a) $\Rightarrow$ (b) is trivial.

(b) $\Rightarrow$ (c) Let $X$ be $\alpha$-basically disconnected, let $G$ be an $\alpha$-open subset of $X$, and let $Z_{1}$ and $Z_{2}$ be disjoint zero-sets of $G$. It suffices to show that $Z_{1}$ and $Z_{2}$ are completely separated in $X$.

By 2.1, $Z_{1}$ and $Z_{2}$ are contained in disjoint $\alpha$-open subsets $P_{1}$ and $P_{2}$ of $X$ respectively. Then $\mathrm{cl}_{X} P_{1}$ and $\mathrm{cl}_{X} P_{2}$ are disjoint closed-and-open subsets of $X$ and hence $Z_{1}$ and $Z_{2}$ are completely separated in $X$.

(c) $\Rightarrow$ (a) By [GJ, 1H.6] it suffices to show that every open subset of $X$ is $C^{*}$-embedded in $X$, and this follows from (c) and the fact that the cozero-sets form a base for $X$.

The following is a generalization of [GJ, 14N.4].

2.3. THEOREM. $A$ space $X$ is an $F_{\alpha}$-space if and only if any two disjoint $\alpha$-open subsets of $X$ are completely separated in $X$.

Proof. If $G$ and $H$ are disjoint $\alpha$-open subsets of the $F_{\alpha}$-space $X$, then the function $f \in C^{*}(G \cup H)$ that is 0 on $G$ and 1 on $H$ has an extension $g \in C(X)$, and $g$ completely separates $G$ and $H$. Conversely, let $G$ be $\alpha$-open in $X$ and let $Z_{1}$ and $Z_{2}$ be disjoint zero-sets of $G$. By $2.1, Z_{1}$ and $Z_{2}$ are contained in disjoint $\alpha$-open subsets of $X$, which are completely separated in $X$ by hypothesis, and hence $G$ is $C^{*}$ embedded in $X$. 
The well-known fact that the $F$-space property is hereditary with respect to $C^{*}$-embedded subsets does not generalize to $F_{\alpha}$-spaces (see 2.6), but we do have the following result:

2.4. Theorem. Let $S$ be dense and $C^{*}$-embedded in the space $T$. Then $S$ is an $F_{\alpha}$-space if and only if $T$ is an $F_{\alpha}$-space.

Proof. Let $S$ be an $F_{\alpha}$-space and let $G$ be $\alpha$-open in $T$. Let $f \in C^{*}(G)$. Since $S \cap G$ is $\alpha$-open in $S, S \cap G$ is $C^{*}$-embedded in $S$ and hence in $T$, and thus $f \mid(S \cap G)$ has an extension $g \in C(T)$. Since $S \cap G$ is dense in $G, g \mid G=f$. Thus $G$ is $C^{*}$-embedded in $T$, and we conclude that $T$ is an $F_{\alpha}$-space.

Conversely, assume that $T$ is an $F_{\alpha}$-space and let $G$ be $\alpha$-open in $S$. Let $Z_{1}$ and $Z_{2}$ be disjoint zero-sets of $G$. By 2.1 there exist disjoint $\alpha$-open subsets $H_{1}$ and $H_{2}$ of $S$ such that $Z_{1} \subset H_{1}$ and $Z_{2} \subset H_{2}$. Since $S$ is $C^{*}$-embedded in $T$, there exist $\alpha$-open subsets $L_{1}$ and $L_{2}$ of $T$ such that $H_{1}=L_{1} \cap S$ and $H_{2}=L_{2} \cap S$, and since $S$ is dense in $T, L_{1}$ and $L_{2}$ are disjoint. By $2.3, L_{1}$ and $L_{2}$ are completely separated in $S$. We conclude that $G$ is $C^{*}$-embedded in $S$.

The following corollary generalizes an important property of $F$-spaces [GJ, 14.25].

2.5. Corollary. $A$ space $X$ is an $F_{\alpha}$-space if and only if $\beta X$ is an $F_{\alpha}$-space.

2.6. RemarK. For $\alpha>\omega_{1}$, a $C^{*}$-embedded subset (in fact, a compact subset) of an $F_{\alpha}$-space need not be an $F_{\alpha}$-space: $\beta \omega$ is extremally disconnected, hence an $F_{\alpha}$-space for all $\alpha$, but $\beta \omega-\omega$ is not basically disconnected [GJ, 6W.3], and so there exists a cardinal $\alpha$ such that $\beta \omega-\omega$ is not an $F_{\alpha}$-space. (The referee of this paper has noted that $\beta \omega-\omega$ is not an $F_{\omega_{2}}$-space since Hausdorff has shown that there is an $\left(\omega_{1}, \omega_{1}\right)$-gap in $\beta \omega-\omega[\mathrm{Ha}, \S 1]$.)

The following result generalizes [GJ, 6M.1].

2.7. Proposition. A space $X$ is $\alpha$-basically disconnected if and only if $\beta X$ is $\alpha$-basically disconnected.

Proof. Let $X$ be $\alpha$-basically disconnected and let $G$ be $\alpha$-open in $\beta X$. Then $G \cap X$ is $\alpha$-open in $X$ and hence $\operatorname{cl}_{\beta X} G=\operatorname{cl}_{\beta X}\left(\mathrm{cl}_{X}(G \cap X)\right)$ is open in $\beta X$. Conversely, assume that $\beta X$ is $\alpha$-basically disconnected and let $H$ be $\alpha$-open in $X$. Then $H=H^{\prime} \cap X$, where $H^{\prime}$ is $\alpha$-open in $\beta X$. Since $\mathrm{cl}_{\beta X} H^{\prime}$ is open in $\beta X, \mathrm{cl}_{X} H=X \cap$ $\mathrm{cl}_{\beta X} H^{\prime}$ is open in $X$.

A collection of pairwise disjoint open subsets of a space $X$ is called a cellular family in $X$. A subset $A$ of $X$ is cellularly embedded in $X$ if every cellular family in $A$ is the restriction to $A$ of a cellular family in $X$. We will use the next proposition in $\S 5$.

2.8. Proposition. If $A$ is cellularly embedded in the $F_{\alpha}$-space $X$, and if $w L(A)<\alpha$, then $A$ is $C^{*}$-embedded in $X$.

Proof. Let $Z_{1}$ and $Z_{2}$ be disjoint zero-sets of $A$. There exist disjoint cozero-sets $P_{1}$ and $P_{2}$ of $A$ and disjoint open sets $U_{1}$ and $U_{2}$ of $X$ such that $Z_{1} \subset P_{1} \subset U_{1}$ and $Z_{2} \subset P_{2} \subset U_{2}$. For each $x \in Z_{1}$ and each $y \in Z_{2}$ pick cozero-sets $P_{x}$ and $Q_{y}$ of $X$ 
with $x \in P_{x} \subset U_{1}$ and $y \in Q_{y} \subset U_{2}$. There exist $I \subset Z_{1}$ and $J \subset Z_{2}$ with $|I|$, $|J|<\alpha$ such that $Z_{1} \subset \mathrm{cl}_{X} \cup_{x \in I} P_{x}$ and $Z_{2} \subset \mathrm{cl}_{X} \cup_{y \in J} Q_{y}$. Since $\cup_{x \in I} P_{x}$ and $\cup_{y \in J} Q_{y}$ are disjoint $\alpha$-open subsets of $X, Z_{1}$ and $Z_{2}$ are completely separated in $X$ and hence $A$ is $C^{*}$-embedded in $X$.

Corollary 2.9 is well known (see [GJ, 14N.1]).

2.9. COROllary. No point of an F-space is the limit of a sequence of distinct points.

3. $F_{\alpha}$-spaces and $\chi_{\alpha}$-remote points. We call a space $X \alpha$-small if $\left|C^{*}(X)\right| \leqslant 2^{\alpha}$ and we call $X$ small if $X$ is $\omega$-small. We denote the Stone-Čech remainder $\beta X-X$ of $X$ by $X^{*}$.

The theorem of Fine and Gillman below (3.1) plays a crucial role in the proof that (a) $\Rightarrow$ (c) in 0.1 .

3.1. TheOREM (FINE AND Gillman [FG, 4.6]) [CH]. If $X$ is a small locally compact o-compact space, then $X^{*}$ has no proper dense $C^{*}$-embedded subspace.

Before stating a consequence of 3.1 , we need the following result.

3.2. Proposition [S $\mathbf{S w}_{\mathbf{1}}, 5.3$ ]. If $F$ is closed in the normal space $X$, then $\mathrm{cl}_{\beta X} F$ is $\alpha^{+}$-closed in $\beta X$ if and only if $\chi(F, X) \leqslant \alpha$.

3.3. Proposition [CH]. If $X$ is a small normal $F$-space and if $p \in X^{*}$, then $p \notin \mathrm{cl}_{\beta X} F$ for every closed nowhere dense subset $F$ of $X$ with $\chi(F, X)=\omega$.

Proof. Suppose $p \in X^{*}$ and $p \in \operatorname{cl}_{\beta X} F$ where $F$ is a closed nowhere dense subset of $X$ with $\chi(F, X)=\omega$. By 3.2, $\mathrm{cl}_{\beta X} F$ is a nowhere dense zero-set of $\beta X$ and hence $\beta X-\operatorname{cl}_{\beta X} F$ is a dense cozero-set of the $F$-space $\beta X$. Then $\mathrm{cl}_{\beta X} F$ is the Stone-Čech remainder of the small locally compact $\sigma$-compact space $\beta X-\operatorname{cl}_{\beta X} F$. By 3.1, then, $\mathrm{cl}_{\beta X} F$ has no proper dense $C^{*}$-embedded subset, which is, since $p \in\left(\operatorname{cl}_{\beta X} F\right)-F$, a contradiction.

What we have, then, is that, under the hypothesis of 3.3, all points of $X^{*}$ are "remote" in some sense. We make this motion precise by defining a point $p \in X^{*}$ to be a $\chi_{\alpha}$-remote point of $X$ if $p \notin \mathrm{cl}_{\beta X} F$ for every closed nowhere dense subset $F$ of $X$ with $\chi(F, X) \leqslant \alpha$. A point $p \in X^{*}$, then, is remote in the sense of $\left[\mathbf{v D}_{2}\right]$ if and only if $p$ is $\chi_{\alpha}$-remote for every cardinal $\alpha$; and if $\chi_{c}(X) \leqslant \alpha$, then for every $p \in X^{*}, p$ is $\chi_{\alpha}$-remote if and only if $p$ is remote.

What we will establish in this paper is that, under a suitable generalization of the hypothesis of $0.1(\mathrm{c})$, no point of $X^{*}$ is $\chi_{\alpha}$-remote. In the case $\alpha=\omega$, then, by 3.3, $X^{*}=\varnothing$.

If $\mathscr{F}$ is a collection of sets, we say that $\mathscr{F}$ has the $\alpha$-intersection property if $\cap \mathscr{F}^{\prime} \neq \varnothing$ for all $\mathscr{F}^{\prime} \subset \mathscr{F}$ with $\left|\mathscr{F}^{\prime}\right|<\alpha$. We set $\beta_{\alpha} X=\{p \in \beta X$ : $p$ has the $\alpha$-intersection property (where points of $\beta X$ are regarded as $z$-ultrafilters on $X$ ). A space $X$ is $\alpha$-pseudocompact if $\beta_{\alpha^{+}} X=\beta X$ [Ke]. Note that the Hewitt realcompactification $v X$ of $X$ is $\beta_{\omega_{1}} X$ and that $X$ is pseudocompact if and only if $X$ is $\omega$-pseudocompact.

A space $X$ is $[\alpha, \beta]$-compact if every open cover $\mathscr{U}$ of $X$ with $|\mathcal{Q}| \leqslant \beta$ has a subcover $\mathcal{U}^{\prime}$ such that $\left|\mathcal{U}^{\prime}\right|<\alpha$. It can be shown (see [Ke, 2.2 and §3]) that if $X$ is $[\omega, \alpha]$-compact, then $X$ is $\alpha$-pseudocompact. 
For our purposes, it turns out that " $\alpha$-pseudocompact" is the appropriate generalization of "countably compact" in $0.1(\mathrm{c})$. The important fact about an $\alpha$-pseudocompact space $X$ for this study is that, since $X$ is $G_{\alpha^{+}}$-dense in $\beta_{\alpha^{+}} X$, if $X$ is $\alpha$-pseudocompact, then every $G_{\alpha^{+}}$-set in $\beta X$ meets $X$.

We are now ready to state the main theorem of this section.

3.4. TheOREM $\left[2^{\alpha}=\alpha^{+}\right]$. If $X$ is an $\alpha$-small, $\alpha$-pseudocompact, normal $F_{\alpha^{+}}$-space, then $X$ has no $\chi_{\alpha}$-remote point.

Before proceeding with the proof of 3.4 , let us note that $0.1(a) \Rightarrow(c)$ is a corollary.

3.5. Corollary (Woods [Wo, $1.1(\mathrm{a})]$ ) $[\mathrm{CH}]$. If $X$ is a small normal countably compact $F$-space, then $X$ is compact.

Proof. By 3.3, every point of $X^{*}$ is $\chi_{\omega}$-remote. Hence by $3.4, X^{*}=\varnothing$.

We postpone the proof of 3.4 until after we have established a generalization of $0.1(a) \Rightarrow(b)$. This we do in a sequence of lemmas in order to show precisely where the hypothesis $2^{\alpha}=\alpha^{+}$is needed.

The easy proof of the first lemma is omitted.

3.6. Lemma. If $L(X) \leqslant \alpha$, and if $G$ is $\alpha^{+}$-open in $X$, then $L(G) \leqslant \alpha$.

The next lemma is a generalization of [FG, 4.1]. The lemma is essentially [CN, 14.1] (except that the latter has an unnecessary regularity hypothesis on $\alpha$ ), and we are therefore omitting the proof. A detailed proof can be found in [S $\mathbf{S} \mathbf{w}_{\mathbf{2}}, \mathbf{1 6}$.10].

3.7. LemMA. Let $X$ be an $F_{\alpha}$-space and let $V=\cup_{\xi<\alpha} V_{\xi}$ be an $\alpha^{+}$-open subset of $X$. If $G \subset V$ and if $G \cap V_{\xi}$ is $\alpha$-open in $V$ for each $\xi<\alpha$, then $G$ is $C^{*}$-embedded in $V$.

The proof of the following lemma uses techniques similar to those of $\left[\mathbf{W o}_{1}, 2.2\right]$.

3.8. Lemma. If $w L(X)=L(X)=\alpha^{+}$, then there exists an $\alpha^{++}$-open subset $V=\cup_{\xi<\alpha^{+}} V_{\xi}$ of $\beta X$ such that $X \subset V$ and there exists a pairwise disjoint collection $\left\{P_{\xi}: \xi<\alpha^{+}\right\}$of cozero-sets of $\beta X$ with each $P_{\xi} \subset V$ and such that for all $\beta<\alpha^{+}$, $V_{\beta} \cap \cup_{\xi<\alpha^{+}} P_{\xi}$ is $\alpha^{+}$-open in $V$.

Proof. Since $w L(X)=\alpha^{+}$, there is an open cover थ of cozero-sets of $X$ such that no subcover of cardinality $\alpha$ has dense union in $X$. Since $L(X)=\alpha^{+}, \mathcal{Q}$ has a subcover $\left\{U_{\xi}: \xi<\alpha^{+}\right\}$of cardinality $\alpha^{+}$. Then each $U_{\xi}=V_{\xi} \cap X$, where $V_{\xi}$ is a cozero-set of $\beta X$. Let $V=\cup_{\xi<\alpha^{+}} V_{\xi}$ and note that $X \subset V$. We will construct, by recursion, a sequence $\left\langle P_{\xi}: \xi<\alpha^{+}\right\rangle$satisfying the following two conditions:

(1) For each $\xi<\alpha^{+}, P_{\xi}$ is a nonempty cozero-set in $\beta X$ and $P_{\xi} \subset V$.

(2) For each $\beta, \xi<\alpha^{+}$, if $\beta<\xi$, then $P_{\xi} \cap\left(P_{\beta} \cup V_{\beta}\right)=\varnothing$.

Let $\xi<\alpha^{+}$and assume that for all $\beta<\xi, P_{\beta}$ has been selected subject to conditions (1) and (2).

We will now show that $V-\mathrm{cl}_{\beta X} \cup_{\beta<\xi} P_{\beta} \neq \varnothing$. Assume the contrary. Since $\cup_{\beta<\xi}\left(P_{\beta} \cup V_{\beta}\right)$ is $\alpha^{+}$-open in $\beta X$, since $\left\{V_{\xi}: \xi<\alpha^{+}\right\}$covers $\cup_{\beta<\xi}\left(P_{\beta} \cup V_{\beta}\right)$ and since $L(\beta X)=\omega \leqslant \alpha$, by 3.6 there exists $I \subset \alpha^{+}$such that $|I| \leqslant \alpha$ and such that 
$\left\{V_{\xi}: \xi \in I\right\}$ covers $\cup_{\beta<\xi}\left(P_{\beta} \cup V_{\beta}\right)$. Then $\cup_{\beta<\xi}\left(P_{\beta} \cup V_{\beta}\right) \subset \cup_{\xi \in I} V_{\xi} \subset V \subset$ $\operatorname{cl}_{\beta X} \cup_{\beta<\xi}\left(P_{\beta} \cup V_{\beta}\right)$, and hence $\cup_{\xi \in I} V_{\xi}$ is dense in $V$. Therefore $\left(\cup_{\xi \in I} V_{\xi}\right) \cap X=$ $\cup_{\xi \in I} U_{\xi}$ is dense in $V \cap X=X$, contradicting the assumption that no subcollection of थ of cardinality $\leqslant \alpha$ has dense union in $X$.

We may therefore select a nonempty cozero-set $P_{\xi}$ of $\beta X$ such that $P_{\xi} \subset V-$ $\mathrm{cl}_{\beta X} \cup_{\beta<\xi}\left(P_{\beta} \cup V_{\beta}\right)$. This completes the recursion.

By conditions (1) and (2), the collection $\left\{P_{\xi}: \xi<\alpha^{+}\right\}$satisfies the requirements of the lemma.

3.9. Lemma. If $w L(X)=L(X)=\alpha^{+}$and if $X$ is an $F_{\alpha^{+}}$-space, then $\left|C^{*}(X)\right| \geqslant 2^{\alpha^{+}}$.

Proof. By 3.8, there exists an $\alpha^{++}$-open subset $V$ of $\beta X$ with $X \subset V$, and there exists $P=\cup_{\xi<\alpha^{+}} P_{\xi}$, a pairwise disjoint collection of cozero-sets of $\beta X$ with $P \subset V$ and such that for all $\xi<\alpha^{+}, V_{\xi} \cap P$ is $\alpha^{+}$-open in $V$. Since $\beta X$ is an $F_{\alpha^{+}}$-space, $P$ is $C^{*}$-embedded in $V$ by 3.7, and, since $X \subset V, V$ is $C^{*}$-embedded in $\beta X$. Then $2^{\alpha^{+}} \leqslant\left|C^{*}(P)\right| \leqslant\left|C^{*}(V)\right| \leqslant\left|C^{*}(\beta X)\right|=\left|C^{*}(X)\right|$.

3.10. TheOrem. If $\left|C^{*}(X)\right| \leqslant \alpha^{+}$and if $X$ is an $F_{\alpha^{+}}$-space, then $w L(X) \leqslant \alpha$.

Proof. If $w L(X) \geqslant \alpha^{+}$, then $w L(X)=L(X)=w(X)=\left|C^{*}(X)\right|=\alpha^{+}$, contradicting 3.9.

3.11. Corollary $\left[2^{\alpha}=\alpha^{+}\right]$. If $X$ is an $\alpha$-small $F_{\alpha^{+}}$-space, then $w L(X) \leqslant \alpha$.

We see in 5.2 that Corollary 3.11 is, in fact, equivalent to $2^{\alpha}=\alpha^{+}$.

We still have some more work to do before we can proceed with the proof of 3.4 . First we need a couple of lemmas.

3.12. Lemma. Let $X$ be a space with $w L(X) \leqslant \alpha$. If $p \in X^{*}$, then there exists a dense $\alpha^{+}$-open subset $V$ of $\beta X$ with $p \notin V$.

Proof. $\beta X-\{p\}$ is open in $\beta X$, and hence $\beta X-\{p\}=\cup \mathcal{U}$, where $\mathscr{Q}$ is a collection of cozero-sets of $\beta X$. Since $X \subset \cup \mathcal{Q}$ and $w L(X) \leqslant \alpha$, there exists $\mathscr{U}^{\prime} \subset \mathcal{U}$ such that $\left|\mathscr{U}^{\prime}\right| \leqslant \alpha$ and such that $\left(\cup \mathcal{U}^{\prime}\right) \cap X$ is dense in $X$. Let $V=\cup \mathcal{U}^{\prime}$. $V$ is a dense $\alpha^{+}$-open subset of $\beta X$ and $p \notin V$.

3.13. LEMMA. If $X$ is an $\alpha$-pseudocompact space and if $V$ is a dense $\alpha^{+}$-open subset of $\beta X$, then $\operatorname{cl}_{\beta X}(X-V)=\beta X-V$.

Proof. Let $p \in \beta X-V$ and let $U$ be an open neighborhood of $p$ in $\beta X$. Since $V$ is $\alpha^{+}$-open in $\beta X, \beta X-V$ is $\alpha^{+}$-closed in $\beta X$ and is thus a $G_{\alpha^{+}}$-set in $\beta X$. Hence $U-V=U \cap(\beta X-V)$ is a $G_{\alpha^{+}}$-set in $\beta X=\beta_{\alpha^{+}} X$, and hence $U-V$ meets $X$. Then clearly $U$ meets $X-V$, which implies that $p \in \operatorname{cl}_{\beta X}(X-V)$. The reverse inclusion is obvious.

The following result is fundamental to the proof of 3.4 .

3.14. THEOREM. If $X$ is a normal $\alpha$-pseudocompact space with $w L(X) \leqslant \alpha$, then no point of $X^{*}$ is a $\chi_{\alpha}$-remote point of $X$. 
Proof. Let $p \in X^{*}$. By 3.12 there exists a dense $\alpha^{+}$-open subset $V$ of $\beta X$ such that $p \notin V$. By 3.13, $\operatorname{cl}_{\beta X}(X-V)=\beta X-V$, and hence $p \in \operatorname{cl}_{\beta X}(X-V)$ and $\mathrm{cl}_{\beta X}(X-V)$ is $\alpha^{+}$-closed in $\beta X$. By 3.2, $\chi(X-V, X) \leqslant \alpha$, which implies that $p$ is not $\chi_{\alpha}$-remote.

We digress in order to give two corollaries of 3.14. (It is known, of course, that Lindelöf pseudocompact spaces are compact. The second corollary of 3.14 answers the question: What property can one add to weakly Lindelöf pseudocompact normal spaces to insure that they are compact?)

3.15. COROllary. Let $\alpha$ be an infinite cardinal. If $X$ is a normal space, then the following are equivalent:

(a) $X$ is compact.

(b) $w L(X) \leqslant \alpha, X$ is $\alpha$-pseudocompact, and every nowhere dense closed subset $F$ of $X$ with $\chi(F, X) \leqslant \alpha$ is compact.

Proof. (a) $\Rightarrow(b)$ is trivial. Assume (b) and suppose that (a) is false. Then there exists $p \in X^{*}$ and, by $3.14, p$ is not $\chi_{\alpha}$-remote. Therefore some nowhere dense closed subset $F$ of $X$ with $\chi(F, X) \leqslant \alpha$ is not compact, a contradiction.

3.16. COROLLARY. If $X$ is a normal space, then the following are equivalent:

(a) $X$ is compact.

(b) $X$ is weakly Lindelö and pseudocompact, and every closed nowhere dense subset $F$ of $X$ with $\chi(F, X)=\omega$ is compact.

3.17. Remarks. (a) To show that the hypothesis of normality is necessary in 3.16 , let $\phi(\mathbf{R})=\left\{p \in \beta \mathbf{R}: p \notin \mathrm{cl}_{\beta \mathbf{R}} A\right.$ for every closed discrete subset $A$ of $\left.\mathbf{R}\right\} .(\phi(\mathbf{R})$ is the set of far points of $\mathbf{R}$. See $\left[\mathbf{v D}_{2}\right]$ for a discussion of $\phi(\mathbf{R})$.) Let $Y=\mathbf{R} \cup \phi(\mathbf{R})$. $Y$ is separable, hence weakly Lindelöf, and pseudocompact $\left[\mathbf{v D}_{2}, \S 17\right]$. We will show that every closed nowhere dense subset of $Y$ with countable character is compact (and is, in fact, contained in $\mathbf{R}$ ).

Let $F \subset Y$ be closed and nowhere dense and let $\left\{U_{n}: n \in \omega\right\}$ be a base for the neighborhoods of $F$ in $Y$. We show first that $F \cap \mathbf{R}$ is compact.

Assume the contrary. Thus $F \cap \mathbf{R}$ is not bounded and hence contains $\left\{x_{n}\right.$ : $n \in \omega\}$, an infinite closed discrete set. There exists a collection $\left\{H_{n}: n \in \omega\right\}$ of open subsets of $\mathbf{R}$ such that for all $n \in \omega, x_{n} \in H_{n} \subset U_{n}$ and such that $\left\{H_{n}: n \in \omega\right\}$ is discrete in $\mathbf{R}$. Since $H_{n}$ is open in $Y$ and since $F$ is nowhere dense, we can pick $y_{n} \in H_{n}-F$ for all $n \in \omega$. Then $A=\left\{y_{n}: n \in \omega\right\}$ is closed discrete in $\mathbf{R}$ and, since no point of $\phi(\mathbf{R})$ is a limit point of $A, A$ is also closed in $Y$. Then $Y-A$ is a neighborhood of $F$ in $Y$ but no $U_{n}$ is contained in $Y-A$, a contradiction.

Next we show that $F \cap \phi(\mathbf{R})$ has countable character in $Y$. Since $F \cap \mathbf{R}$ is compact and $\phi(\mathbf{R})$ is closed in $Y, F \cap \mathbf{R}$ has a closed neighborhood $H$ in $Y$ such that $H \cap(F \cap \phi(\mathbf{R}))=\varnothing$. Then $\left\{U_{n}-H: n \in \omega\right\}$ is a base for the neighborhoods of $F \cap \phi(\mathbf{R})$ in $Y$.

Finally, we show that no nonempty subset of $\phi(\mathbf{R})$ has countable character in $Y$ and conclude that $F=F \cap \mathbf{R}$ and is therefore compact. Let $\varnothing \neq A \subset \phi(\mathbf{R})$ and let $\left\{H_{n}: n \in \omega\right\}$ be a decreasing neighborhood base for $A$ in $Y$. For all $n \in \omega$, pick 
$y_{n} \in H_{n} \cap \mathbf{R}$ and consider $K=\operatorname{cl}_{\mathbf{R}}\left\{y_{n}: n \in \omega\right\}$. If $K$ is compact, then $Y-K$ is open, $A \subset Y-K$, but for all $n \in \omega, H_{n} \not \subset Y-K$, a contradiction. If $K$ is not compact, $\left\{x_{n}: n \in \omega\right\}$ is not bounded and therefore contains an infinite subset $D=\left\{x_{n k}\right.$ : $k \in \omega\}$ which is closed discrete in $\mathbf{R}$. Again no point of $\phi(\mathbf{R})$ is a limit point of $D$ and therefore $Y-D$ is a neighborhood of $A$ in $Y$. Since $\left\{H_{n}: n \in \omega\right\}$ is a decreasing neighborhood base for $A$ in $Y,\left\{H_{n k}: k \in \omega\right\}$ is also a neighborhood base for $A$ in $Y$, but no $H_{n k}$ is contained in $Y-D$. This contradiction completes the proof.

(b) We show next that, in the implication (b) $\Rightarrow$ (a) of 3.16 , none of the hypotheses of (b) can be omitted.

The ordinal space $\omega_{1}$ is normal and pseudocompact and every closed nowhere dense subset of $\omega_{1}$ with countable character is compact. Thus the hypothesis " $X$ is weakly Lindelöf" cannot be omitted. To see that the hypothesis of pseudocompactness cannot be omitted, we note that the ordinal space $\omega$ is normal and Lindelöf and contains no nonempty nowhere dense closed set. The last example shows that the hypothesis on closed nowhere dense sets cannot be omitted from 3.16(b): Let $\kappa$ be an uncountable cardinal, and for each $\xi<\kappa$, let $X_{\xi}=\{0,1\}$. Let $X=\Pi_{\xi<\kappa} X_{\xi}$ and let $Y=\left\{x \in X: x_{\xi}=1\right.$ for at most countably many $\left.\xi<\kappa\right\}$. ( $Y$ is a " $\Sigma$-product" of the family $\left\langle X_{\xi}: \xi<\kappa\right\rangle$. See [Co] and [En, 2.7.13] for further details.) By [Co, Theorem 1], $Y$ is normal, and by [Co, Theorem 2], $v Y=X=\beta Y$, and hence $Y$ is pseudocompact but not compact. Moreover, by [Co, Proposition 3], $Y$ has a dense Lindelöf subspace and is therefore weakly Lindelöf. (We are grateful to E. K. van Douwen for calling [Co] to our attention.)

We are now ready to prove 3.4 .

Proof OF 3.4. Let $x$ be an $\alpha$-small $\alpha$-pseudocompact normal $F_{\alpha^{+}}$-space. By 3.11, $w L(X) \leqslant \alpha$ and hence by $3.14, X$ has no $\chi_{\alpha}$-remote point.

The statement of the following theorem is also equivalent to $2^{\alpha}=\alpha^{+}$(see 5.2).

3.18. THEOREM $\left[2^{\alpha}=\alpha^{+}\right]$. If $X$ is an $\alpha$-small $F_{\alpha^{+}}$-space with $\chi(F, X) \leqslant \alpha$ for every $\alpha^{+}$-closed nowhere dense subset $F$ of $X$, then $\beta_{\alpha^{+}} X-X$ contains no $\chi_{\alpha^{-}}$-remote point of $X$.

Proof. Let $p \in \beta_{\alpha^{+}} X-X$. By 3.11 and 3.12 there exists a dense $\alpha^{+}$-open subset $V$ of $\beta X$ with $p \notin V$. Then $\beta X-V$ is $\alpha^{+}$-closed in $\beta X$ and hence is a $G_{\alpha^{+}}$-set in $\beta X$. Since $p \in \beta_{\alpha}, X,(\beta X-V) \cap U$ meets $X$ for every neighborhood $U$ of $p$ in $\beta X$, and hence $p \in \operatorname{cl}_{\beta X}(X-V)$. Since $\chi(X-V, X) \leqslant \alpha, p$ is not $\chi_{\alpha}$-remote.

3.19. CoRollary $[\mathrm{CH}]$. If $X$ is a small normal F-space with $\chi_{n z}(X)=\omega$, then $X$ is realcompact.

Proof. Let $p \in v X-X$. By 3.18, $p \in \mathrm{cl}_{\beta X} F$ for some closed nowhere dense set $F$ of $X$ with $\chi(F, X)=\omega$, contradicting 3.3.

3.20. RemarKs. (a) 3.19 is equivalent to $\mathrm{CH}$ (see 5.3(b)).

(b) In $\left[\mathbf{S w}_{1}\right]$ we show that if $X$ is countably compact, then $\chi(Z, X)=\omega$ for every zero-set $Z$ of $X$, and hence $\chi_{n z}(X)=\omega$. Note then that $0.1(\mathrm{a}) \Rightarrow(\mathrm{c})$ is a corollary of 3.19. Note also that for $p \in \beta \omega-\omega, 3.19$ shows that, under $\mathrm{CH}, \beta \omega-\{p\}$ is not 
normal. (See [Wa, 7.3 and 7.4] for a proof that $\mathrm{CH}$ implies that $\omega^{*}-\{p\}$ (and hence $\beta \omega-\{p\})$ is not normal.)

(c) In [Te], Terada shows that no point of $v X-X$ is remote, provided only that every collection of disjoint open subsets of $X$ has Ulam-nonmeasurable cardinality. We will see later, however, that for every $\alpha$, there is a space $\Phi_{\alpha}$ such that $v \Phi_{\alpha}-\Phi_{\alpha}$ contains a $\chi_{\alpha}$-remote point (see 4.26).

4. Generalization of van Douwen's space $\Phi$. In this section we define, for every infinite cardinal $\alpha$, a space $\Phi_{\alpha}$ that is normal, almost compact, noncompact, $\alpha^{+}$-basically disconnected (hence an $F_{\alpha^{+}}$-space), [ $\left.\omega, \alpha^{+}\right]$-compact (hence $\alpha$-pseudocompact) and such that $\chi\left(F, \Phi_{\alpha}\right) \leqslant \alpha$ for every $\alpha^{+}$-closed nowhere dense subset $F$ of $\Phi_{\alpha}$. We shall also show that $w L\left(\Phi_{\alpha}\right)>\alpha$, that there exists a point in $\Phi_{\alpha}^{*}$, hence in $\beta_{\alpha^{+}} \Phi_{\alpha}-\Phi_{\alpha}$, that is $\chi_{\alpha^{-}}$-remote, and that, if $2^{\alpha} \neq \alpha^{+}$, then $\Phi_{\alpha}$ is $\alpha$-small. Hence if $2^{\alpha} \neq \alpha^{+}$, then $\Phi_{\alpha}$ is an example of a space for which the statements of 3.4, 3.11 and 3.18 fail. The conclusion then is that the statements of 3.4, 3.11 and 3.18 are each equivalent to the segment $2^{\alpha}=\alpha^{+}$of GCH (see 5.2).

$\Phi_{\alpha}$ is a straightforward cardinal generalization of van Douwen's space $\Phi$ of $\left[\mathbf{v D}_{1}\right]$ (in fact, $\Phi_{\omega}=\Phi$ ), and many of the techniques used in proving that $\Phi_{\alpha}$ has the properties listed above are those of $\left[\mathbf{v D}_{1}\right]$.

The space $\Phi_{\alpha}$ is defined as a subspace of $\beta B_{\alpha}$, where $B_{\alpha}$ is a cardinal generalization of the space $P$ of $\left[\mathbf{v D}_{1}\right]$ ( $P$ is also described in [GJ, 9L]). $B_{\alpha}$ is a subspace of $\alpha^{++}+1$, and is defined as follows:

$$
B_{\alpha}=\left\{\xi \leqslant \alpha^{++}: \xi \text { is a successor ordinal or } \operatorname{cf}(\xi) \in\left\{\alpha^{+}, \alpha^{++}\right\}\right\} .
$$

We now turn to the properties of $B_{\alpha}$ that will be used in discussing $\Phi_{\alpha}$.

4.1. Proposition. If $A$ is an initial segment of $B_{\alpha}$ and if $f \in C(A)$, then $f$ is constant on some neighborhood of each point in $A$.

Proof. The result follows from the fact that countable intersections of open sets are open in $B_{\alpha}$.

\subsection{Proposition. Every $\alpha^{+}$-open subset of $B_{\alpha}$ is closed in $B_{\alpha}$.}

Proof. Let $G=\cup_{\xi<\alpha} P_{\xi}$, where each $P_{\xi}$ is the cozero-set of $f_{\xi} \in C\left(B_{\alpha}\right)$ and suppose there exists $\beta \in\left(\mathrm{cl}_{B_{\alpha}} G\right)-G$. Then $f_{\xi}(\beta)=0$ for all $\xi<\alpha$. $\beta$ is not a successor ordinal since $\beta \notin G$, and hence $\operatorname{cf}(\beta) \geqslant \alpha^{+}$. By 4 .1, for each $\xi<\alpha$, there exists $\nu_{\xi}<\beta$ such that $\left(\nu_{\xi}, \beta\right] \cap B_{\alpha} \subset f_{\xi}^{-1}(0)$. Let $\nu=\sup \left\{\nu_{\xi}: \xi<\alpha\right\}$. Then $\nu<\beta$ and $(\nu, \beta] \cap B_{\alpha} \subset f_{\xi}^{-1}(0)$ for all $\xi<\alpha$. Hence $(\nu, \beta] \cap G=\varnothing$, which contradicts the assumption that $\beta \in \mathrm{cl}_{B_{\alpha}} G$.

4.3. Corollary. $B_{\alpha}$ is $\alpha^{+}$-basically disconnected, and hence an $F_{\alpha^{+}}$-space.

4.4. Proposition. $L\left(B_{\alpha}\right)=\alpha$.

Proof. Let $\mathscr{Q}$ be an open cover of $B_{\alpha}$. There exists $U \in \mathcal{Q}$ and there exists $\beta<\alpha^{++}$such that $\left(\beta, \alpha^{++}\right] \cap B_{\alpha} \subset U \in \mathcal{Q}$. Hence it suffices to show that

$$
L\left([0, \beta] \cap B_{\alpha}\right) \leqslant \alpha \quad \text { if } \beta<\alpha^{++} .
$$


Assume then that $\mathcal{Q}$ is an open cover of $[0, \beta] \cap B_{\alpha}$. We may assume that Q $=\left\{\mathscr{Q}_{\xi}: \xi<\alpha^{+}\right\}$and that each $U_{\xi} \subset[0, \beta] \cap B_{\alpha}$. For all $\xi<\alpha^{+}$, let $V_{\xi}=U_{\xi}$ $\cup_{\delta<\xi} U_{\delta}$, and let $I=\left\{\xi<\alpha^{+}: V_{\xi} \neq \varnothing\right\}$. It suffices to show that $|I| \leqslant \alpha$.

Assume that $|I|=\alpha^{+}$and pick $\nu_{\xi} \in V_{\xi}$ for all $\xi \in I$. Let $\nu=\sup \left\{\nu_{\xi}: \xi \in I\right\}$. Then $\nu \leqslant \beta$ and $\operatorname{cf}(\nu)=\alpha^{+}$and hence there exists $\delta<\alpha^{+}$and there exists $\lambda<\nu$ such that $(\lambda, \nu] \cap B_{\alpha} \subset U_{\delta}$. Then for all $\eta>\delta,(\lambda, \nu] \cap B_{\alpha} \cap V_{\eta}=\varnothing$, a contradiction. Thus (*) holds, which completes the proof.

4.5. Proposition. $w\left(B_{\alpha}\right)=\alpha^{++}$.

Proof. $\alpha^{++}=\chi\left(B_{\alpha}\right) \leqslant w\left(B_{\alpha}\right) \leqslant\left|B_{\alpha}\right| \cdot \chi\left(B_{\alpha}\right)=\alpha^{++} \cdot \alpha^{++}=\alpha^{++}$.

For a space $X$, we set $\mathrm{CO}(X)=\{A: A$ is closed-and-open in $X\}$.

We call a space $X$ strongly zero-dimensional if every pair of disjoint zero-sets of $X$ can be separated by disjoint closed-and-open subsets of $X$ [En, 6.2.4].

We turn now to a sequence of propositions leading to a computation of $w\left(\beta B_{\alpha}\right)$.

4.6. Proposition. $B_{\alpha}$ is strongly zero-dimensional.

Proof. This is immediate from 4.2 .

The next two propositions are no doubt well known, but no references are known to the author.

4.7. Proposition. For any space $X,|\mathrm{CO}(X)| \leqslant w(X)^{L(X)}$.

Proof. Let $\mathscr{B}$ be a base for $X$ with $|\mathscr{B}| \leqslant w(X)$. Define $\Psi$ : $\operatorname{CO}(X) \rightarrow\left\{\mathscr{B}^{\prime} \subset \mathscr{B}\right.$ : $\left.\left|\mathscr{B}^{\prime}\right| \leqslant L(X)\right\}$ by $\Psi(A)=\mathscr{Q}_{A}$ if $A=\cup \mathscr{Q}_{A}$. Then $\Psi$ is injective and $\mid\left\{\mathscr{B}^{\prime} \subset \mathscr{B}\right.$ : $\left.\left|\mathscr{B}^{\prime}\right| \leqslant L(X)\right\} \mid \leqslant w(X)^{L(X)}$.

4.8. Proposition. For any space $X,|\mathrm{CO}(X)|=|\mathrm{CO}(\beta X)|$.

Proof. Define $\Psi: \operatorname{CO}(X) \rightarrow \operatorname{CO}(\beta X)$ by $\Psi(U)=\operatorname{cl}_{\beta X} U$. Then $\Psi$ is bijective.

The following two cardinal equalities will be used in subsequent calculations. Proofs can easily be given using [Mo, 22.5, 22.6, 22.13 and 22.14].

4.9. Proposition. (a) $\left(\alpha^{++}\right)^{\alpha}=\alpha^{++} \cdot 2^{\alpha}$.

(b) $\left(\alpha^{++} \cdot 2^{\alpha}\right)^{\alpha^{++}}=2^{\alpha^{++}}$.

4.10. Proposition. $\left|\operatorname{CO}\left(B_{\alpha}\right)\right|=\alpha^{++} \cdot 2^{\alpha}$, and hence $\left|\operatorname{CO}\left(\beta B_{\alpha}\right)\right|=\alpha^{++} \cdot 2^{\alpha}$.

Proof. $\left|\operatorname{CO}\left(B_{\alpha}\right)\right| \leqslant\left(\alpha^{++}\right)^{\alpha}=\alpha^{++} \cdot 2^{\alpha}$ by $4.4,4.5,4.7$ and 4.9(a). Let $\mathscr{Q}=\{\{\xi\}: \xi$ is a successor ordinal and $\xi>\alpha\}$ and let $\mathscr{B}=\left\{A: A \subset[0, \alpha) \cap B_{\alpha}\right\}$. Then $\left|\operatorname{CO}\left(B_{\alpha}\right)\right| \geqslant|\mathscr{Q}| \cdot|\Re|=\alpha^{++} \cdot 2^{\alpha}$, and hence $\left|\operatorname{CO}\left(\beta B_{\alpha}\right)\right|=\alpha^{++} \cdot 2^{\alpha}$ by 4.8 .

4.11. Proposition. $w\left(\beta B_{\alpha}\right)=\alpha^{++} \cdot 2^{\alpha}$.

Proof. Since $B_{\alpha}$ is strongly zero-dimensional, so is $\beta B_{\alpha}$ [En, 6.2.12] and therefore $w\left(\beta B_{\alpha}\right)=\left|\mathrm{CO}\left(\beta B_{\alpha}\right)\right|$ by [CN, 2.24]. The result then follows from 4.10.

We prove two more results about $B_{\alpha}$ that will be needed later.

4.12. Proposition. If $G$ is $\alpha^{+}$-open and dense in $\beta B_{\alpha}$, then $B_{\alpha} \subset G$. 
Proof. If $G$ is $\alpha^{+}$-open and dense in $\beta B_{\alpha}$, then $G \cap B_{\alpha}$ is $\alpha^{+}$-open and dense in $B_{\alpha}$. By 4.2, $G \cap B_{\alpha}$ is closed in $B_{\alpha}$, and hence $G \cap B_{\alpha}=B_{\alpha}$.

4.13. Proposition. Every real-valued continuous function on $B_{\alpha}-\left\{\alpha^{++}\right\}$is constant on a tail of $B_{\alpha}-\left\{\alpha^{++}\right\}$, and hence $B_{\alpha}-\left\{\alpha^{++}\right\}$is $C$-embedded in $B_{\alpha}$.

Proof. Let $f \in C\left(B_{\alpha}-\left\{\alpha^{++}\right\}\right)$. Suppose $f$ is not constant on a tail of $B_{\alpha}-$ $\left\{\alpha^{++}\right\}$. We can then pick recursively two sequences $\left\langle\beta_{\xi}: \xi<\alpha^{+}\right\rangle$and $\left\langle\nu_{\xi}: \xi<\alpha^{+}\right\rangle$ such that $f\left(\beta_{\xi}\right) \neq f\left(\nu_{\xi}\right)$ for all $\xi<\alpha^{+}$and such that if $\delta<\xi$, then $\beta_{\delta}<\nu_{\delta}<\beta_{\xi}$.

Let $\nu=\sup \left\{\beta_{\xi}: \xi<\alpha^{+}\right\}=\sup \left\{\nu_{\xi}: \xi<\alpha^{+}\right\}$. Then $\nu \in B_{\alpha}-\left\{\alpha^{++}\right\}$, but $f$ is not constant on a neighborhood of $\nu$, contradicting 4.1.

We now define the space $\Phi_{\alpha}$ as follows: $\Phi_{\alpha}=\beta B_{\alpha}-\left\{\alpha^{++}\right\}$.

The remainder of this section is devoted to showing that $\Phi_{\alpha}$ has the desired properties.

4.14. Proposition. $\beta \Phi_{\alpha}=\beta B_{\alpha}$, and hence $\Phi_{\alpha}$ is almost compact but not compact.

Proof. $\Phi_{\alpha}$ is clearly dense in $\beta B_{\alpha}$, and since, by $4.13, B_{\alpha}-\left\{\alpha^{++}\right\}$is $C$-embedded in $B_{\alpha}, \Phi_{\alpha}$ is $C^{*}$-embedded in $\beta B_{\alpha}$.

4.15. Proposition. $\Phi_{\alpha}$ is collectionwise normal.

Proof. We show that every pair of noncompact closed subsets of $\Phi_{\alpha}$ meet. Let $F$ and $K$ be closed subsets of $\Phi_{\alpha}$ with $\alpha^{++} \in \operatorname{cl}_{\beta \Phi_{\alpha}} F \cap \operatorname{cl}_{\beta \Phi_{\alpha}} K$. We will pick, recursively, four sequences $\left\langle x_{\xi}: \xi<\alpha^{+}\right\rangle,\left\langle y_{\xi}: \xi<\alpha^{+}\right\rangle,\left\langle\nu_{\xi}: \xi<\alpha^{+}\right\rangle$and $\left\langle\beta_{\xi}: \xi<\alpha^{+}\right\rangle$ subject to the following conditions:

(1) For all $\xi<\alpha^{+}$,

$$
x_{\xi} \in F \cap \operatorname{cl}_{\beta \Phi_{\alpha}}\left(\left(\beta_{\xi}, \nu_{\xi}\right] \cap B_{\alpha}\right) \quad \text { and } \quad y_{\xi} \in K \cap \operatorname{cl}_{\beta \Phi_{\alpha}}\left(\left(\beta_{\xi}, \nu_{\xi}\right] \cap B_{\alpha}\right) .
$$

(2) If $\delta<\xi<\alpha^{+}$, then $\beta_{\delta}<\nu_{\delta} \leqslant \beta_{\xi}<\alpha^{++}$.

Let $\xi<\alpha^{+}$and assume that for all $\delta<\xi, x_{\delta}, y_{\delta}, \nu_{\delta}$ and $\beta_{\delta}$ have been selected satisfying (1) and (2). Let $\beta_{\xi}=\sup \left\{\nu_{\delta}: \delta<\xi\right\}$. Pick

$$
x_{\xi} \in F \cap \operatorname{cl}_{\beta \Phi_{\alpha}}\left(\left(\beta_{\xi}, \alpha^{++}\right] \cap B_{\alpha}\right) \quad \text { and } y_{\xi} \in K \cap \operatorname{cl}_{\beta \Phi_{\alpha}}\left(\left(\beta_{\xi}, \alpha^{++}\right] \cap B_{\alpha}\right) .
$$

Pick $\nu_{\xi}$ so that $x_{\xi}, y_{\xi} \notin \mathrm{cl}_{\beta \Phi_{\alpha}}\left(\left(\nu_{\xi}, \alpha^{++}\right] \cap B_{\alpha}\right)$. This completes the recursion.

Let $\nu=\sup \left\{\beta_{\xi}: \xi<\alpha^{+}\right\}=\sup \left\{\nu_{\xi}: \xi<\alpha^{+}\right\}$. Since $\operatorname{cf}(\nu)=\alpha^{+}, \nu \in \Phi_{\alpha}$. Also

$$
\nu \in \operatorname{cl}_{\Phi_{\alpha}}\left\{x_{\xi}: \xi<\alpha^{+}\right\} \subset F \text { and } \nu \in \operatorname{cl}_{\Phi_{\alpha}}\left\{y_{\xi}: \xi<\alpha^{+}\right\} \subset K .
$$

Thus $F$ and $K$ meet which proves that $\Phi_{\alpha}$ is normal. Since $\Phi_{\alpha}$ is almost compact, $\Phi_{\alpha}$ is collectionwise normal.

4.16. Proposition. $\Phi_{\alpha}$ is $\alpha^{+}$-basically disconnected and hence an $F_{\alpha^{+}}$-space.

Proof. By 4.3, $B_{\alpha}$ is $\alpha^{+}$-basically disconnected. The result then follows from 2.7 and 4.14.

4.17. Proposition. $w L\left(\Phi_{\alpha}\right)=\alpha^{++}$. 
Proof. Since $B_{\alpha}-\left\{\alpha^{++}\right\}$is dense in $\Phi_{\alpha}, w L\left(\Phi_{\alpha}\right) \leqslant\left|B_{\alpha}-\left\{\alpha^{++}\right\}\right|=\alpha^{++}$. To see that $w L\left(\Phi_{\alpha}\right) \geqslant \alpha^{++}$, we note that $\mathscr{U}=\left\{\beta B_{\alpha}-\mathrm{cl}_{\beta B_{\alpha}}\left(\left(\xi, \alpha^{++}\right] \cap B_{\alpha}\right): \xi<\alpha^{++}\right\}$is an open cover of $\Phi_{\alpha}$ of cardinality $\alpha^{++}$. No subcover of $\mathscr{U}$ of smaller cardinality has dense union in $\Phi_{\alpha}$.

4.18. Proposition. If $F$ is $\alpha^{+}$-closed and nowhere dense in $\Phi_{\alpha}$, then $F$ is $\alpha^{+}$-closed in $\beta B_{\alpha}$ and hence $F$ is compact.

Proof. We may write $F=\bigcap_{\xi<\alpha}\left(\Phi_{\alpha}-P_{\xi}\right)$. where each $P_{\xi}$ is a cozero-set in $\beta \Phi_{\alpha}=\beta B_{\alpha}$. Then $\cup_{\xi<\alpha} P_{\xi}$ is dense and $\alpha^{+}$-open in $\beta B_{\alpha}$. which implies by 4.12 , that $B_{\alpha} \subset \cup_{\xi<\alpha} P_{\xi}$. Hence $\alpha^{++} \in \cup_{\xi<\alpha} P_{\xi}$, and so $F=\bigcap_{\xi<\alpha}\left(\beta B_{\alpha}-P_{\xi}\right)$. an $\alpha^{+}$-closed subset of $\beta B_{\alpha}$.

4.19. Proposition. $\chi\left(F, \Phi_{\alpha}\right) \leqslant \alpha$ for every nowhere dense $\alpha^{+}$-closed subset $F$ of $\Phi_{\alpha}$.

Proof. By 4.18, $F$ is $\alpha^{+}$-closed in $\beta B_{\alpha}$ and hence $\chi\left(F, \Phi_{\alpha}\right) \leqslant \chi\left(F, \beta B_{\alpha}\right)=$ $\psi\left(F, \beta B_{\alpha}\right) \leqslant \alpha$.

The next group of propositions leads to the conclusion that $\Phi_{\alpha}$ is $\alpha^{+}$pseudocompact. We will show, in fact, that $\Phi_{\alpha}$ is $\left[\omega, \alpha^{+}\right]$-compact.

A point $p \in X$ is a complete accumulation point of a subset $A$ of $X$ if for every neighborhood $U$ of $p$ in $X,|U \cap A|=|A|$.

4.20. Lemma [En, 3.12.1]. A space $X$ is compact if and only if every infinite subset of $X$ has a complete accumulation point in $X$.

4.21. Proposition. If $A \subset \Phi_{\alpha}$ and if $|A| \leqslant \alpha^{+}$, then $\alpha^{++}$is not a limit point of $A$ in $\beta B_{\alpha}$.

Proof. Since $\operatorname{cf}\left(\alpha^{++}\right)>\alpha^{+}, \alpha^{++} \notin \mathrm{cl}_{\beta B_{\alpha}} A$ if $|A| \leqslant \alpha^{+}$.

4.22. Proposition. $\Phi_{\alpha}$ is $\left[\omega, \alpha^{+}\right]$-compact.

Proof. Let $\mathscr{U}$ be an open cover of $\Phi_{\alpha}$ such that $|\mathscr{U}| \leqslant \alpha^{+}$, and assume that $\mathscr{Q}$ has no finite subcover. Choose $\mathcal{U}^{\prime} \subset \mathcal{U}$ of minimal cardinality such that $\mathcal{U}^{\prime}$ covers $\Phi_{\alpha}$. Write $\mathscr{U}^{\prime}=\left\{U_{\xi}: \xi<\kappa\right\}$ with $\kappa \leqslant \alpha^{+}$, and for each $\xi<\kappa$, let $V_{\xi}=U_{\xi}-\cup_{\beta<\xi} U_{\beta}$. Let $I=\left\{\xi<\kappa: V_{\xi} \neq \varnothing\right\}$, and note that $\Phi_{\alpha}=\cup_{\xi \in I} U_{\xi}$. By the minimality of $\kappa$, $|I|=\kappa$. For each $\xi \in I$, choose $x_{\xi} \in V_{\xi}$, and let $A=\left\{x_{\xi}: \xi \in I\right\}$. Clearly $|A|=\kappa \geqslant \omega$, and hence $A$ has a complete accumulation point $p \in \beta B_{\alpha}$. By $4.21, p \neq \alpha^{++}$, and hence $p \in \Phi_{\alpha}$. Then $p \in U_{\beta}$ for some $\beta \in I$, and therefore $\left|U_{\beta} \cap A\right|=|A|=\kappa$. But for all $\xi \in I$ with $\xi>\beta$, we have $x_{\xi} \notin U_{\beta}$ and hence $\left|U_{\beta} \cap A\right| \leqslant|\beta|<\kappa$, a contradiction.

4.23. CoRollary. $\Phi_{\alpha}$ is $\alpha^{+}$-pseudocompact, hence $\alpha$-pseudocompact.

In order to calculate $\left|C^{*}\left(\Phi_{\alpha}\right)\right|$, we need the following result from elsewhere. (We discuss this result in more detail in $\$ 5$.)

4.24. Theorem [CoHa, 2.2]. For any space $X,|C(X)| \leqslant w(X)^{w L(X)}$.

4.25. Proposition. $\left|C\left(\Phi_{\alpha}\right)\right|=\alpha^{++} \cdot 2^{\alpha}$. 
Proof. It suffices to calculate $\left|C^{*}\left(\beta B_{\alpha}\right)\right|$. By 4.11, 4.24 and $4.9,\left|C^{*}\left(\beta B_{\alpha}\right)\right| \leqslant$ $\left(\alpha^{++} \cdot 2^{\alpha}\right)^{\omega} \leqslant\left(\alpha^{++} \cdot 2^{\alpha}\right)^{\alpha}=\alpha^{++} \cdot 2^{\alpha}$, and, since the characteristic function of a closed-and-open set is continuous, $\left|C^{*}\left(\beta B_{\alpha}\right)\right| \geqslant\left|\operatorname{CO}\left(\beta B_{\alpha}\right)\right|=\alpha^{++} \cdot 2^{\alpha}$ by 4.10 .

We need one last result about $\Phi_{\alpha}$.

4.26. Proposition. $\alpha^{++}$is a $\chi_{\alpha^{-}}$-remote point of $\Phi_{\alpha}$.

Proof. Let $F$ be a closed nowhere dense subset of $\Phi_{\alpha}$ with $\chi\left(F, \Phi_{\alpha}\right) \leqslant \alpha$. By 3.2, $\operatorname{cl}_{\beta \Phi_{\alpha}} F$ is $\alpha^{+}$-closed in $\beta \Phi_{\alpha}$, which implies that $F=\Phi_{\alpha} \cap \operatorname{cl}_{\beta \Phi_{\alpha}} F$ is $\alpha^{+}$-closed in $\Phi_{\alpha}$. By 4.18, $F$ is compact, and hence $\alpha^{++} \notin \mathrm{cl}_{\beta \Phi_{\alpha}} F$.

5. Topological characterizations of cardinal equalities. As noted in 4.24, Comfort and Hager prove in [CoHa, 2.2] that, for every space $X,|C(X)| \leqslant w(X)^{w L(X)}$, and in [CoHa, 5.5], they give an example of a space $X$ such that $|C(X)|<w(X)^{w L(X)}$. In [vDZ], van Douwen and Zhou observe that, for every cardinal $\lambda$ such that $\lambda^{\omega}=\lambda$, the ordinal space $\lambda$ has the property that $|C(\lambda)|=\lambda$ while $w(\lambda)^{w L(\lambda)}>\lambda$.

What we now show is that, for every infinite cardinal $\alpha,\left|C\left(\Phi_{\alpha}\right)\right|<w\left(\Phi_{\alpha}\right)^{w L\left(\Phi_{\alpha}\right)}$ if and only if $2^{\alpha}<2^{\alpha^{++}}$. In fact, we have the following topological characterizations of the cardinal equality $2^{\alpha}=2^{\alpha^{++}}$.

5.1. THEOREM. If $\alpha$ is an infinite cardinal, then the following are equivalent:

(a) $2^{\alpha}=2^{\alpha^{++}}$.

(b) If $X$ is any space with $d(X) \leqslant \alpha^{++}$and if $X$ has either a discrete $C^{*}$-embedded subspace of cardinality $\alpha$ or at least $2^{\alpha}$ closed-and-open subsets, then $|C(X)|=$ $w(X)^{w L(X)}$.

(c) If $X$ is an $F_{\alpha^{+}}$-space with $d(X) \leqslant \alpha^{++}$, and if $X$ has a discrete cellularly embedded subset of cardinality $\alpha$, then $|C(X)|=w(X)^{w L(X)}$.

(d) $\left|C\left(\Phi_{\alpha}\right)\right|=w\left(\Phi_{\alpha}\right)^{w L\left(\Phi_{\alpha}\right)}$.

Proof. (a) $\Rightarrow$ (b) Under either hypothesis on $X,\left|C^{*}(X)\right| \geqslant 2^{\alpha}$. Then, by [CoHa 2.2] and [En, 1.5.6], $2^{\alpha} \leqslant\left|C^{*}(X)\right|=|C(X)| \leqslant w(X)^{w L(X)} \leqslant\left(2^{d(X)}\right)^{d(X)} \leqslant 2^{\alpha^{++}}=2^{\alpha}$.

(b) $\Rightarrow$ (c) This is immediate from 2.8 .

(c) $\Rightarrow$ (d) Since $B_{\alpha}-\left\{\alpha^{++}\right\}$is dense in $\Phi_{\alpha}, d\left(\Phi_{\alpha}\right) \leqslant \alpha^{++}$. Moreover, the set $S=[0, \alpha) \cap B_{\alpha}$ is cellularly embedded in $\Phi_{\alpha}$ and $|S|=\alpha$.

(d) $\Rightarrow$ (a) If $2^{\alpha}<2^{\alpha^{++}}$, then $\left|C\left(\Phi_{\alpha}\right)\right|=\alpha^{++} \cdot 2^{\alpha}<2^{\alpha^{++}}=\left(\alpha^{++} \cdot 2^{\alpha}\right)^{\alpha^{++}}=$ $w\left(\Phi_{\alpha}\right)^{w L\left(\Phi_{\alpha}\right)}$.

As a summary of $\$ \S 3$ and 4 , we now present several topological characterizations of the segment $2^{\alpha}=\alpha^{+}$of GCH. It should again be noted that 5.2 , in the case $\alpha=\omega$, is due partly to Woods [Wo, 2.2$]$ and $\left[\mathbf{W o}_{\mathbf{2}}, 1.1(\mathrm{a})\right]$ and partly to van Douwen $\left[\mathbf{v D}_{1}\right]$.

5.2. THEOREM. If $\alpha$ is an infinite cardinal, then the following are equivalent:

(a) $2^{\alpha}=\alpha^{+}$.

(b) If $X$ is an $\alpha$-small $F_{\alpha^{+}}$-space, then $w L(X) \leqslant \alpha$.

(c) If $X$ is an $\alpha$-small, $\alpha$-pseudocompact, normal $F_{\alpha^{+}}-$space, then $X$ has no $\chi_{\alpha^{-}}$-remote point. 
(d) If $X$ is an $\alpha$-small $F_{\alpha^{+}}$-space such that $\chi(F, X) \leqslant \alpha$ for every $\alpha^{+}$-closed nowhere dense subset $F$ of $X$, then $\beta_{\alpha^{+}} X-X$ contains no $\chi_{\alpha^{-}}$-remote point.

Proof. (a) $\Rightarrow(\mathrm{b})$ is 3.11 ; (a) $\Rightarrow$ (c) is 3.4 ; and (a) $\Rightarrow(d)$ is 3.18 .

Now assume that (a) is false. Then $\left|C^{*}\left(\Phi_{\alpha}\right)\right|=\alpha^{++} \cdot 2^{\alpha}=2^{\alpha}$ and hence $\Phi_{\alpha}$ is $\alpha$-small. By $4.16, \Phi_{\alpha}$ is an $F_{\alpha^{+}}$-space; by $4.15, \Phi_{\alpha}$ is normal; by $4.23, \Phi_{\alpha}$ is $\alpha$-pseudocompact; and by $4.19, \chi\left(F, \Phi_{\alpha}\right) \leqslant \alpha$ for every $\alpha^{+}$-closed nowhere dense subset $F$ of $X$. Then (b) is false by 4.17 while (c) and (d) are false by 4.26 (since $\left.\alpha^{++} \in \beta_{\alpha^{+}} \Phi_{\alpha}-\Phi_{\alpha}\right)$.

5.3. REMARKs. (a) Since by $4.22, \Phi_{\alpha}$ is $[\omega, \alpha]$-compact, the hypothesis " $\alpha$-pseudocompact" can be replaced by " $[\omega, \alpha]$-compact" in $5.2(\mathrm{c})$.

(b) If $\neg \mathrm{CH}$, then $\Phi_{\omega}$ is a small normal $F$-space and $\chi_{n z}\left(\Phi_{\omega}\right)=\omega$ by 4.19 . Since $\Phi_{\omega}$ is countably compact but not compact, $\Phi_{\omega}$ is not realcompact and therefore the statement of 3.19 is equivalent to $\mathrm{CH}$.

\section{REFERENCES}

[CoHa] W. W. Comfort and A. W. Hager, Estimates for the number of real-valued continuous functions, Trans. Amer. Math. Soc. 150 (1970), 619-631.

[CN] W. W. Comfort and S. Negrepontis, The theory of ultrafilters, Die Grundlehren der Math. Wissenschaften, Band 211, Springer-Verlag, New York-Heidelberg-Berlin, 1974.

[Co] H. H. Corson, Normality in subsets of product spaces, Amer. J. Math. 81 (1959), 785-796.

$\left[\mathrm{vD}_{1}\right]$ E. K. van Douwen, $A$ basically disconnected normal space $\Phi$ with $|\beta \Phi-\Phi|=1$, Canad. J. Math. 31 (1979), 911-914.

$\left[\mathbf{v D}_{2}\right] \ldots$ Remote points, Dissertationes Math. 188, PWN, Warsaw, 1981.

[vDZ] E. K. van Douwen and H. X. Zhou, The number of cozero-sets is an w-power, Topology Appl. (to appear).

[Do] A. Dow, On F-spaces and $F^{\prime}$-spaces, preprint.

[En] R. Engelking, General topology, PWN, Warsaw, 1975; English transl., PWN, Warsaw, 1977.

[FG] N. J. Fine and L. Gillman, Extension of continuous functions in $\beta N$, Bull. Amer. Math. Soc. 66 (1960), 376-381.

[GJ] L. Gillman and M. Jerison, Rings of continuous functions, University Series in Higher Math., Van Nostrand, Princeton, N. J., 1960.

[Ha] F. Hausdorff, Summen von $\aleph_{1}$ Mengen, Fund. Math. 26 (1936), 241-255.

[Ke] J. F. Kennison, m-pseudocompactness, Trans. Amer. Math. Soc. 104 (1962), 436-442.

[Mo] J. D. Monk, Introduction to set theory, McGraw-Hill, New York, 1969.

[NL] C. Neville and S. Lloyd, $\boldsymbol{\aleph}$-projective spaces, Illinois J. Math. 25 (1981), 159-168.

[Sw $\left.\mathbf{S w}_{1}\right]$ M. A. Swardson, The character of certain closed sets, Canad. J. Math. (to appear).

$\left[\mathbf{S w}_{\mathbf{2}}\right]$, Generalizations of F-spaces and some topological characterizations of the generalized continuum hypothesis, Ph.D. thesis, Ohio University, 1981.

$\left[\mathrm{Sw}_{3}\right]$, Some topological characterizations of the generalized continuum hypothesis, Lecture Notes in Pure and Appl. Math., Dekker, New York (to appear).

[Te] T. Terada, On remote points in $v X-X$, Proc. Amer. Math. Soc. 77 (1979), 264-266.

[Wa] R. C. Walker, The Stone-Čech compactification, Ergebnisse der Math. und ihrer Grenzgebiete, Band 83, Springer-Verlag, New York-Heidelberg-Berlin, 1974.

[Wo $\mathbf{W}_{1}$ ] R. G. Woods, Characterizations of some $C^{*}$-embedded subspaces of $\beta N$, Pacific J. Math. 65 (1976), 573-579.

$\left[\mathbf{W o}_{2}\right]$ The structure of small normal F-spaces, Topology Proc. 1 (1976), 173-179.

Department of Mathematics, Ohio University, Athens, Ohio 45701 